Strong optical coupling between semiconductor microdisk lasers : From whispering gallery modes to collective modes

\title{
Roiz, Mikhail
}

2020-05-07

Roiz , M , Monakhov , A , Kunitsyna , E , Yakovlev , Y , Teissier , R \& Baranov , A 2020 , ' Strong optical coupling between semiconductor microdisk lasers : From whispering gallery modes to collective modes ' , Journal of Applied Physics, vol. 127 , no. 17 , 173105 . https://doi.org/10.1063/5.0004

http://hdl.handle.net/10138/329687

https://doi.org/10.1063/5.0004273

unspecified

publishedVersion

Downloaded from Helda, University of Helsinki institutional repository.

This is an electronic reprint of the original article.

This reprint may differ from the original in pagination and typographic detail.

Please cite the original version. 


\section{Strong optical coupling between}

semiconductor microdisk lasers: From whispering gallery modes to collective modes

Cite as: J. Appl. Phys. 127, 173105 (2020); https://doi.org/10.1063/5.0004273

Submitted: 11 February 2020 . Accepted: 23 April 2020 . Published Online: 07 May 2020

Mikhail Roiz (D), Andrey Monakhov (D), Ekaterina Kunitsyna, Yury Yakovlev, Roland Teissier, and Alexei Baranov (iD)
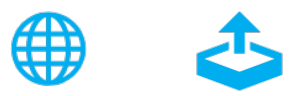

\section{ARTICLES YOU MAY BE INTERESTED IN}

Janus nanoparticle synthesis: Overview, recent developments, and applications

Journal of Applied Physics 127, 170902 (2020); https://doi.org/10.1063/5.0003329

Spin-orbit-torque magnonics

Journal of Applied Physics 127, 170901 (2020); https://doi.org/10.1063/5.0007095

Excitonic effects on photophysical processes of polymeric carbon nitride

Journal of Applied Physics 127, 170903 (2020); https://doi.org/10.1063/5.0005825

\section{Lock-in Amplifiers up to $600 \mathrm{MHz}$}
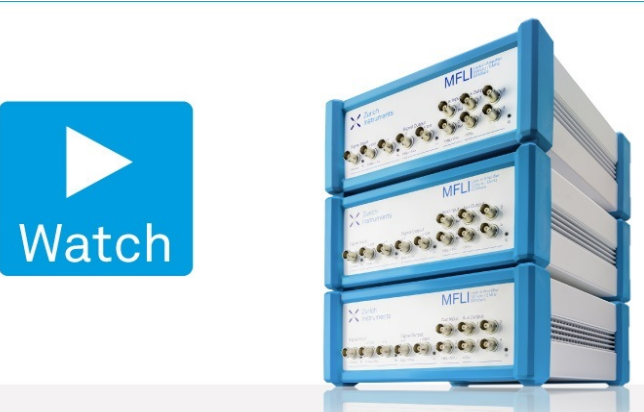


\title{
Strong optical coupling between semiconductor microdisk lasers: From whispering gallery modes to collective modes
}

Cite as: J. Appl. Phys. 127, 173105 (2020); doi: 10.1063/5.0004273

Submitted: 11 February 2020 . Accepted: 23 April 2020 .

Published Online: 7 May 2020

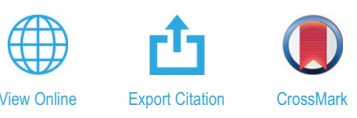

Mikhail Roiz,, ${ }^{1,2, a)}$ (D) Andrey Monakhov, ${ }^{7}$ (D) Ekaterina Kunitsyna, ${ }^{7}$ Yury Yakovlev, ${ }^{7}$ Roland Teissier, ${ }^{3}$ and Alexei Baranov ${ }^{3}$ (D)

\author{
AFFILIATIONS \\ ${ }^{1}$ loffe Institute, St. Petersburg, 194021 Russia \\ ${ }^{2}$ Department of Chemistry, University of Helsinki, P.O. Box 55, FI-00014 Helsinki, Finland \\ ${ }^{3}$ Institut d'Electronique du Sud (IES), Université Montpellier 2, 34095 Montpellier, France
}

${ }^{a)}$ Author to whom correspondence should be addressed: mikhail.roiz@helsinki.fi

\begin{abstract}
In the present paper, we investigate a special type of optical coupling between two electrically pumped semiconductor whispering gallery mode lasers. We fabricate microdisk lasers with a conjunction between the laser cavities, making the coupling stronger. Our experimental results combined with numerical calculations demonstrate a variety of mode families that exist in such systems. Furthermore, we confirm the generation of resonance modes that exist only under simultaneous pumping conditions. These modes are attributed to the collective mode family, which can lead to a single mode operation.
\end{abstract}

Published under license by AIP Publishing. https://doi.org/10.1063/5.0004273

\section{INTRODUCTION}

Whispering Gallery Modes (WGMs) have attracted major attention in the last two decades since they possess unique properties for different applications in science and technology. In particular, this phenomenon has been thoroughly investigated in passive and active optical microresonators. ${ }^{1,2}$ In such microresonators, WGMs have a high quality factor (Q-factor) and a small mode volume. These two important properties make it possible to confine light in a tiny volume inside the optical cavity for a very long time, thus enhancing light-matter interactions. ${ }^{1}$ For instance, in the case of passive microresonators made of third order nonlinear crystals, WGMs allow for the efficient generation of high repetition rate $(>10 \mathrm{GHz})$ Optical Frequency Combs. ${ }^{3}$ In active media, WGMs open up certain avenues for the fabrication of low threshold lasers. The low threshold lasing has been demonstrated in semiconductor WGM-lasers with electri$\mathrm{cal}^{4}$ and optical pumping ${ }^{5,6}$ as well as in highly doped crystalline microresonators with an optical pumping. ${ }^{7}$ In addition, due to the evanescent-field profile of WGMs outside optical cavities, WGMs are extremely sensitive to environmental changes, making them promising for sensing applications in biology and medicine.
Recently, a new platform based on coupled WGM microresonators has drawn a lot of interest. ${ }^{9}$ It has been theoretically predicted and experimentally observed ${ }^{10-12}$ that coupled microresonators can form so-called "Collective Modes" (CMs) or "super modes." These coupled microresonator systems are usually called Photonic Molecules (PMs), but in order to avoid misunderstanding, we should point out that a different physical phenomenon based on bound photon pairs and triplets that form in gas clouds ${ }^{13}$ also has the same term "photonic molecules," although it has no connection to the topic of this paper. Not only do coupled microresonators allow for the exploration of fundamental physical phenomena owing to their resemblance to real molecules, but they also open up opportunities for highly sensitive sensing applications. The latter is possible because the CMs are even more sensitive to the environmental distortions than single WGM cavities. ${ }^{14}$ Moreover, it has been experimentally shown that PMs can possess bounding and anti-bounding states of the cavity modes, ${ }^{15}$ mode splitting, ${ }^{16}$ and different effects due to clockwise and counterclockwise mode interactions. ${ }^{17}$ In addition, single mode lasing was demonstrated in two size-mismatched coupled WGM cavities based on the Vernier effect, which could also be used for a variety of applications. ${ }^{18}$ Thus, we believe that the 
platform based on coupled WGM cavities is very promising from various points of views.

All the above-mentioned physical phenomena have been found in coupled cavities with weak optical coupling, which means that the modes of the cavities are coupled through a small gap between the cavities. That is, usually called evanescent field coupling and such systems can be theoretically described using coupled mode theory or perturbation theory, thus, in some special cases, one can have approximate analytical solutions. ${ }^{17}$ In this paper, we present a different platform of the coupled WGM cavities-strongly coupled WGM-lasers. The strong optical coupling is achieved by the introduction of a deliberately made conjunction between the optical resonators such that the light can be directly transferred from one optical cavity to another. This system has been demonstrated using two coupled electrically pumped semiconductor WGM-lasers exhibiting the formation of CMs as well as a single mode lasing regime. Please note that we use the term "strongly coupled WGM-lasers" not to underline a high coupling efficiency that could be the case for evanescent field coupling, but rather to emphasize the fact that the generated radiation can be transferred from one optical cavity to another without leaving the resonator, thus the two resonators cannot be considered as two separate units. In such a case, it is rather challenging to obtain even an approximate analytical solution, which implies to use numerical calculations. In this paper, we intend to shed some light on the problem of strongly coupled WGM-lasers presenting new experimental observations, as well as numerical calculations that help to get a better understanding of the electromagnetic field distribution in such devices.

\section{FABRICATION OF LASER SAMPLES AND EXPERIMENTAL SETUP}

The studied WGM-lasers are based on quantum well heterostructure GaInAsSb/AlGaAsSb designed for emission near $2.28 \mu \mathrm{m}$ wavelength at room temperature. The structure was grown by molecular beam epitaxy in a Riber 412 solid source machine. The WGM-laser samples were fabricated using the wet etching process. In order to achieve the strong optical coupling, we use a special photomask, which makes it possible to form a conjunction between the laser cavities during the etching process (see Fig. 1). We tested many different samples of such coupled WGM-lasers and found out that equal disks with $10 \mu \mathrm{m}$ long and $15-20 \mu \mathrm{m}$ wide conjunction in between demonstrated the best performance in terms of $\mathrm{CM}$ generation. We have also tested samples of strongly coupled WGM-lasers with size-mismatched disks. For a more detailed description of the material fabrication, please see Ref. 19. It should be mentioned that "equal cavities" mean that the cavities are equal within the accuracy of our fabrication process. The accuracy was of the order of $2 \mu \mathrm{m}$. This inaccuracy is enough to shift the absolute position of a spectral line on the value of the order of the energy distance between spectral lines. Below, we use "equal cavities" and "equal disks" in this sense.
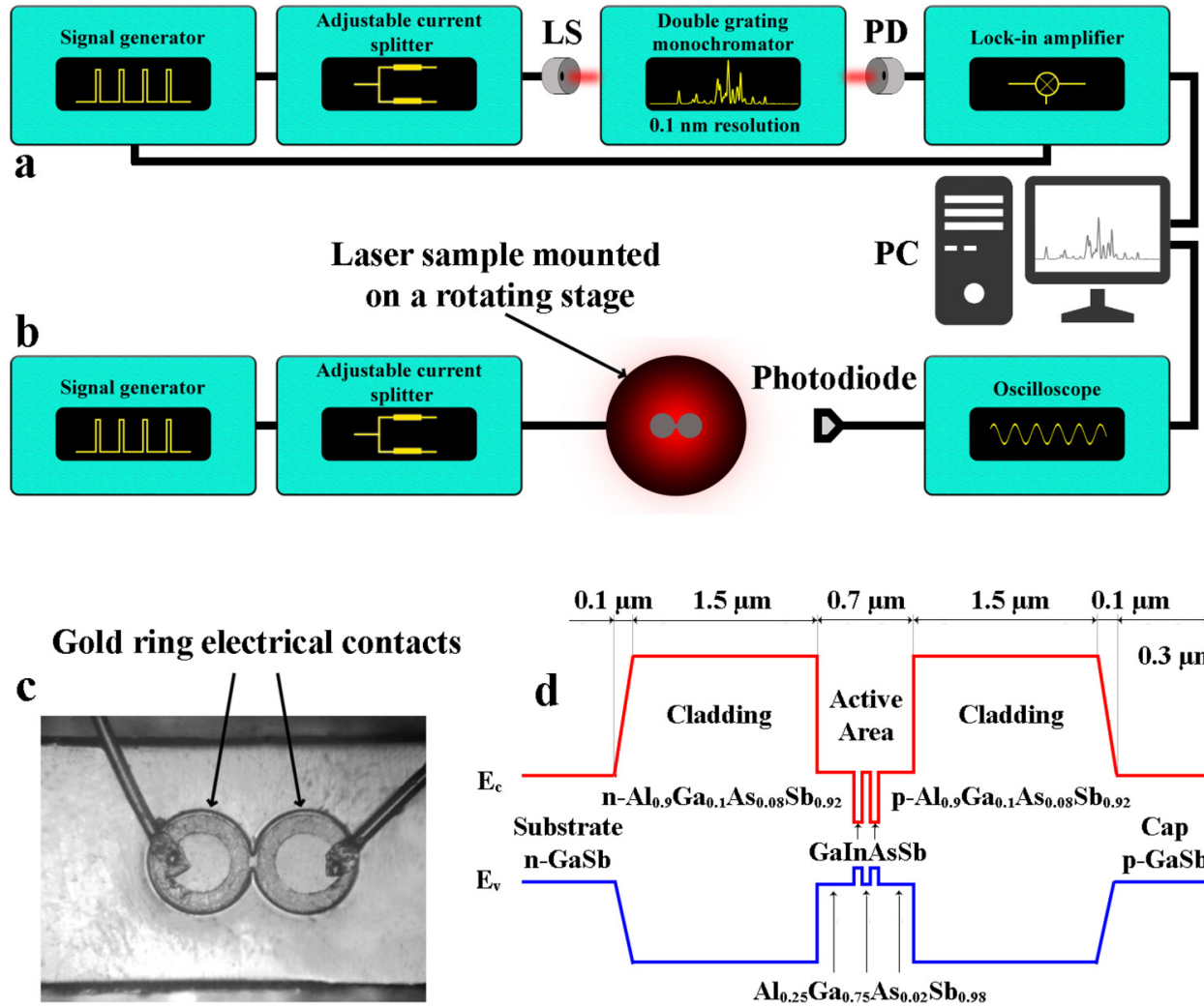

FIG. 1. Block diagram of the experimental setup for spectra (a) and directional diagram (b) measurements; photograph of a strongly coupled WGM-laser sample (c); energy band diagram of the quantum well heterostructure (d); LS-laser sample mounted on a parabolic reflector, PDphotodiode mounted on a parabolic reflector, $\mathrm{PC}$-personal computer. 
In Figs. 1(a) and 1(b), one can see the schematic block diagrams for the measurements of emission spectra and directional diagrams, respectively. We use pulsed electrical pumping with the pulse duration of $2.7 \mu$ s at $30 \mathrm{kHz}$ frequency in order to minimize the thermal effects. This keeps the laser lines at the same relative positions, thus reducing the effect of thermal shift in the whole range of pumping currents. The spectra were measured using parabolic reflectors that collect the radiation from the laser samples and direct it to a monochromator with two diffraction gratings. Please note that the laser samples emit light in all directions if they are not mounted on parabolic reflectors, which is indicated as a red glow around the sample in Fig. 1(b). All coupled WGM-laser samples have two gold ring electrical contacts [see Fig. 1(c)]. Figure 1 (d) shows the energy band diagram of the quantum well heterostructure. It is also worth noting that the average power of our lasers is $0.5-1 \mathrm{~mW}$ measured by a pyroelectric power meter so it includes the spontaneous emission as well.

\section{RESULTS}

\section{A. Single disk WGM-lasers}

First, it is important to verify whether it is possible to generate WGMs in a single microdisk laser using the etching technique that we apply for the coupled WGM-lasers. We design our lasers such that the thickness of the active region $[0.7 \mu \mathrm{m}$, see Fig. $1(\mathrm{~d})]$ is of the order of one wavelength inside the material $(\sim 0.64 \mu \mathrm{m})$. This geometry ensures that we have only first order modes in the polar direction ( $\mathrm{z}$ axis that is perpendicular to the surface of the active region). Figure 2(a) demonstrates the emission spectra of a single electrically pumped WGM-laser with a $200 \mu \mathrm{m}$ radius. It is clear that the periodic structure we observe corresponds to the first order WGMs with a $12 \AA$ Free Spectral Range (FSR). This value is in a good agreement with the calculations of FSR for first order WGMs in a $200 \mu \mathrm{m}$ radius disk using the simple formula

$$
\Delta \lambda=\frac{\lambda^{2}}{2 \pi R n},
$$

where $\Delta \lambda$ is the FSR, $\lambda$ is the wavelength (assuming that the disk is much larger than the wavelength, such that $\Delta \lambda \ll \lambda), R$ is the disk radius, and $n$ is the refractive index of the material ( 3.4 in our case).
Expression (1) results from the analytical solution for a single WGM disk resonator with the perfect metal boundary condition. The analytical solution is well-known for this case, ${ }^{20}$ and formula (1) can be easily derived from the asymptote of the first root of the Bessel function. Moreover, it is verified that formula (1) can also be applied as an approximation of FSR in dielectric disk resonators. ${ }^{20}$

In Fig. 2(b), one can see the Finite Element Method (FEM) simulations of the first order TE WGM calculated for a single disk resonator using perfect metal boundary conditions on the cavity wall. This simulation is done just as a reference point for further consideration. We have tested different disk radii $(50,100$, and $200 \mu \mathrm{m}$ ) in our simulations for both single and coupled WGM resonators and verified that indeed it is possible to find the same mode families in all these cases, which are going to be discussed further in the text. The first order WGMs have the highest Q-factor and stability, thus they are the most efficient modes for the laser to operate on. It has been theoretically investigated by Dontsov et al. that if we introduce a small defect that is of the order of one wavelength inside the cavity, the overall mode structure will not change much, but instead of traveling WGMs the standing WGMs appear. $^{21}$ An entirely different situation comes into play once we introduce a defect in the system that is much larger than the wavelength inside the cavity. The conjunction between the laser cavities would play the role of such a defect. Let us consider this case in more detail.

\section{B. Separately pumped coupled WGM-lasers}

On the next step of our FEM simulations, we introduced the second disk that is connected to the first disk through a conjunction that acts as a large defect in the system. We first consider the case when the pumping current is applied to the first resonator only, and the second resonator absorbs the radiation that is generated by the first resonator. For that purpose, we introduce an imaginary part of the refractive index in the second cavity.

Our simulations demonstrate that the introduction of both the conjunction and the second cavity leads to a significant change in the overall mode structure. Instead of regular WGMs of a single disk laser [see Fig. 2(b)], we now have to consider different modes-leaky and quasi-WGMs. In Fig. 3(a), one can see an example of a leaky mode that resembles a WGM shown in Fig. 2(b), but rather $\mathbf{a}$

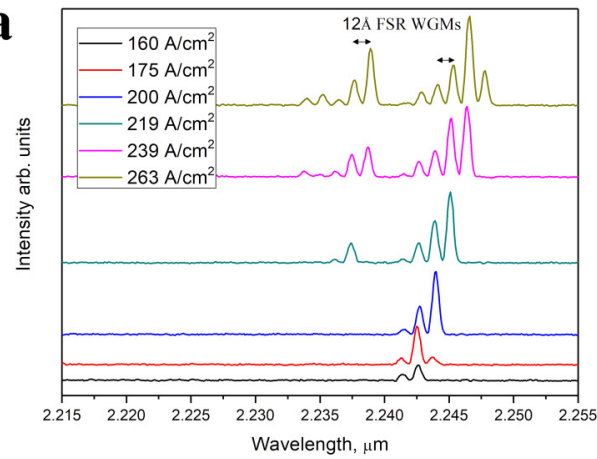

b

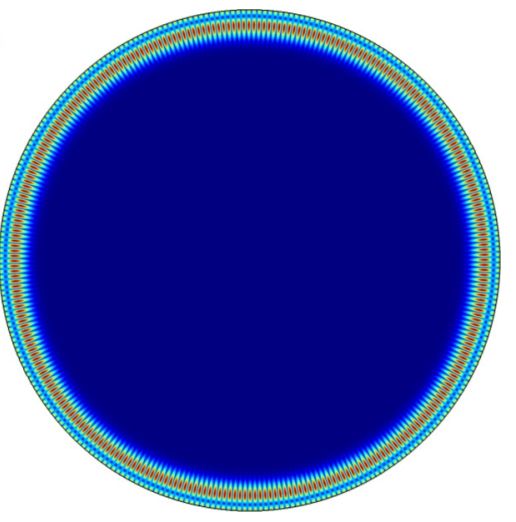

FIG. 2. (a) Emission spectra of a single electrically pumped disk laser with $200 \mu \mathrm{m}$ radius, all the spectra are obtained well above the laser threshold; (b) FEM simulation of the first order TE WGM for a single microdisk. 
a

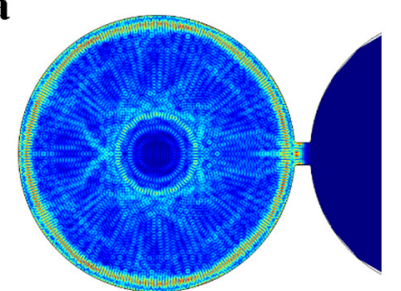

b

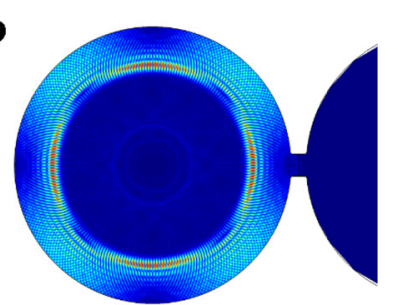

c

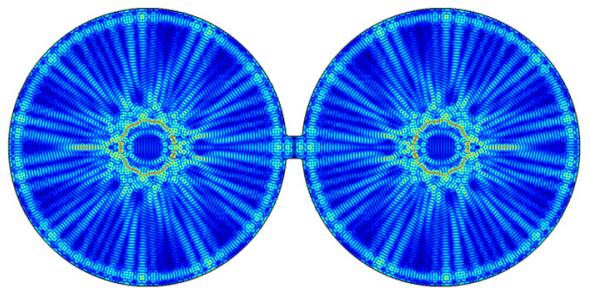

d

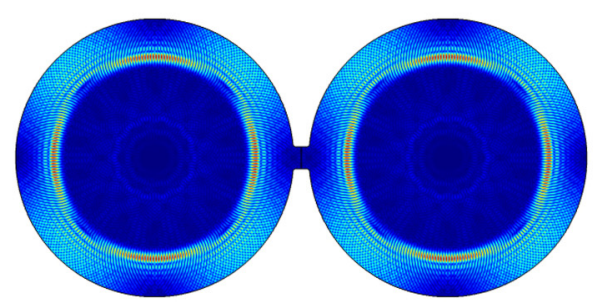

$\mathbf{e}$

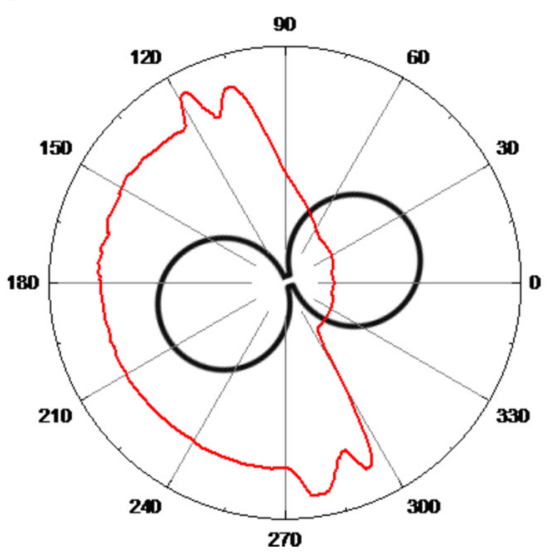

FIG. 3. Results of the FEM simulations (a) leaky quasi-WGM of the strongly coupled WGM-lasers; (b) stable quasi-WGM in the case when only one disk is pumped; (c) collective mode of the strong coupled WGM-lasers when pumped simultaneously; (d) quasi-WGM of the first family; and (e) directional emission diagram of the coupled microdisk WGM-lasers.

distorted, and thus having a much lower Q-factor for two reasons. First, this mode propagates close to the cavity rim and easily leaks into the absorption area. This leads to a dramatic increase in the imaginary part of the calculated eigenfrequency and the lasing condition $\mathrm{KG}=1$, where $\mathrm{G}$ is the optical gain and $\mathrm{K}$ is the feedback factor, fulfilled at much higher optical gains in comparison to the mode shown in Fig. 2(b). The second reason is that the mode experiences scattering losses in the conjunction itself. It can be seen in the experimental directional diagram of the laser emission as two distinct peaks of radiation on both sides of the conjunction [see Fig. 3(e)]. These losses are not taken into account in our simulations (lack of computational resources), since we applied perfect metal boundary conditions on the cavity wall. It should be noted that the directional diagram shows how the laser emission is distributed around the system when only one laser [left in Fig. 3(e)] is pumped. Due to the reasons mentioned above, the lowest order WGMs are not exited in the system. Instead, the most efficient modes of the cavity are higher order quasi-WGMs. ${ }^{22}$ Quasi-WGMs can be thought as WGMs with slightly modified optical paths. The optical path of quasi-WGMs is not fully circular, but rather polygonal. ${ }^{23}$ One can see an example of such a mode in Fig. 3(b). The effective optical path of these modes lies within the first cavity such that the mode minimizes its losses in the conjunction and the second cavity, and thus the imaginary part of the eigenfrequency is close to zero. It is worth noting that, for the quasi-WGMs, formula (1) does not work due to the fact that they cannot be described by a single Bessel function. This leads to the discrepancy in the FSRs on the emission spectra that we observe experimentally, and, in most cases, there is no clear FSR when the lasers are pumped separately.

\section{Simultaneously pumped coupled WGM-lasers}

Let us now consider the case when both lasers are pumped simultaneously. For the sake of computational simplicity, we applied the periodic boundary condition at the very center of the conjunction, such that it separates the system into two symmetric parts, thus all the obtained solutions are symmetric as well. Here, we can distinguish two main mode families. The first mode family consists of those stable quasi-WGMs that we get when only one laser is pumped. In this case, we have weakly interacting lasers that can be considered in the borders of the evanescent field coupling model. ${ }^{17}$ One can see an example of such a mode in Fig. 3(d), which is basically the same solution as in Fig. 3(b), but now obtained for two lasers simultaneously. For the weakly interacting lasers, the resulting spectrum should be just a sum of the two spectra for the lasers being pumped separately, and we can confirm this behavior experimentally. In Fig. 4(a), one can see a set of measured emission spectra of a sample of strongly coupled WGM-lasers, which was associated with a name "sample 1." This particular sample has equal disk radii of $100 \mu \mathrm{m}$. It is clear that the resulting spectrum of simultaneous pumping [Fig. 4(a), 3] is just a sum of the spectra of separately pumped lasers [Fig. 4(a), 1, 2]. It is worth noting that there is anyway one additional mode that exists only when both lasers are pumped, indicating the fact that some interaction between the cavities exists, but it is very weak. This is a usual behavior for the laser samples with a narrow conjunction. When the width of the conjunction is less than $10 \mu \mathrm{m}$, the lasers tend to operate on the stable quasi-WGMs and do not interact with one another. If we increase the width of the conjunction to $15 \mu \mathrm{m}$ or more, the coupling between the cavities becomes substantial and another mode family can emerge.

The second mode family can be considered as the family of collective modes [see Fig. 3(c)]. These modes only appear in the FEM simulations when we disable the absorption in one of the cavities and allow both cavities to freely transfer electromagnetic radiation through the conjunction. Interestingly, these modes have an extremely complex mode structure, and, more importantly, very large effective mode area in the cavity. This makes them more 

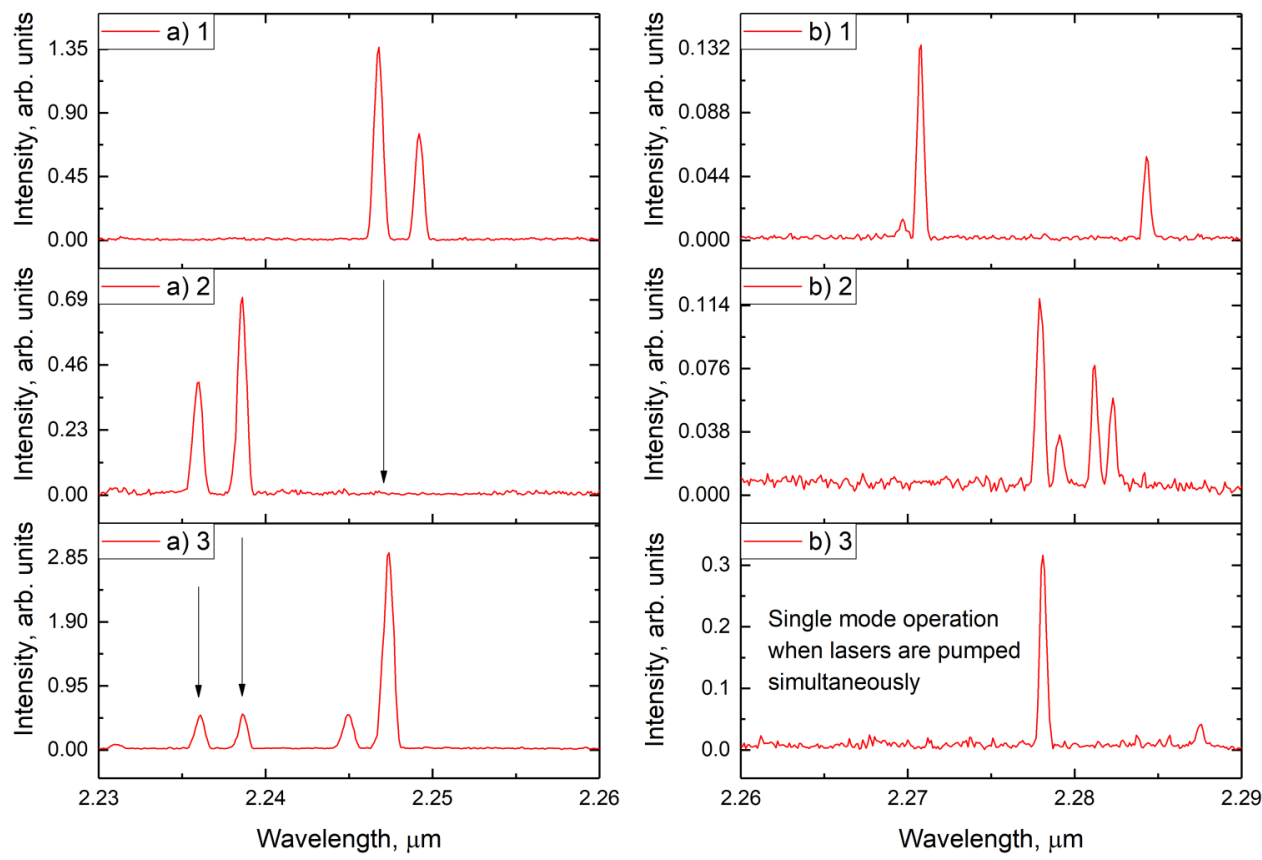

FIG. 4. (a) Measured emission spectra of sample 1 with $100 \mu \mathrm{m}$ equal radii when only the first laser is pumped (1), only the second one (2) and both lasers pumped simultaneously (3); (b) same for sample 2 with $200 \mu \mathrm{m}$ equal radii exhibiting the single mode operation; (c) same for sample 3 with $200 \mu \mathrm{m} \quad$ unintentionally sizemismatched or deformed disks; (d) same for sample 4 with intentionally size-mismatched $100 \mu \mathrm{m}$ and $85 \mu \mathrm{m}$ disk radii; all the spectra are obtained when the pump current density is kept the same for each laser-250 A/cm ${ }^{2}$, it is well above the laser threshold.
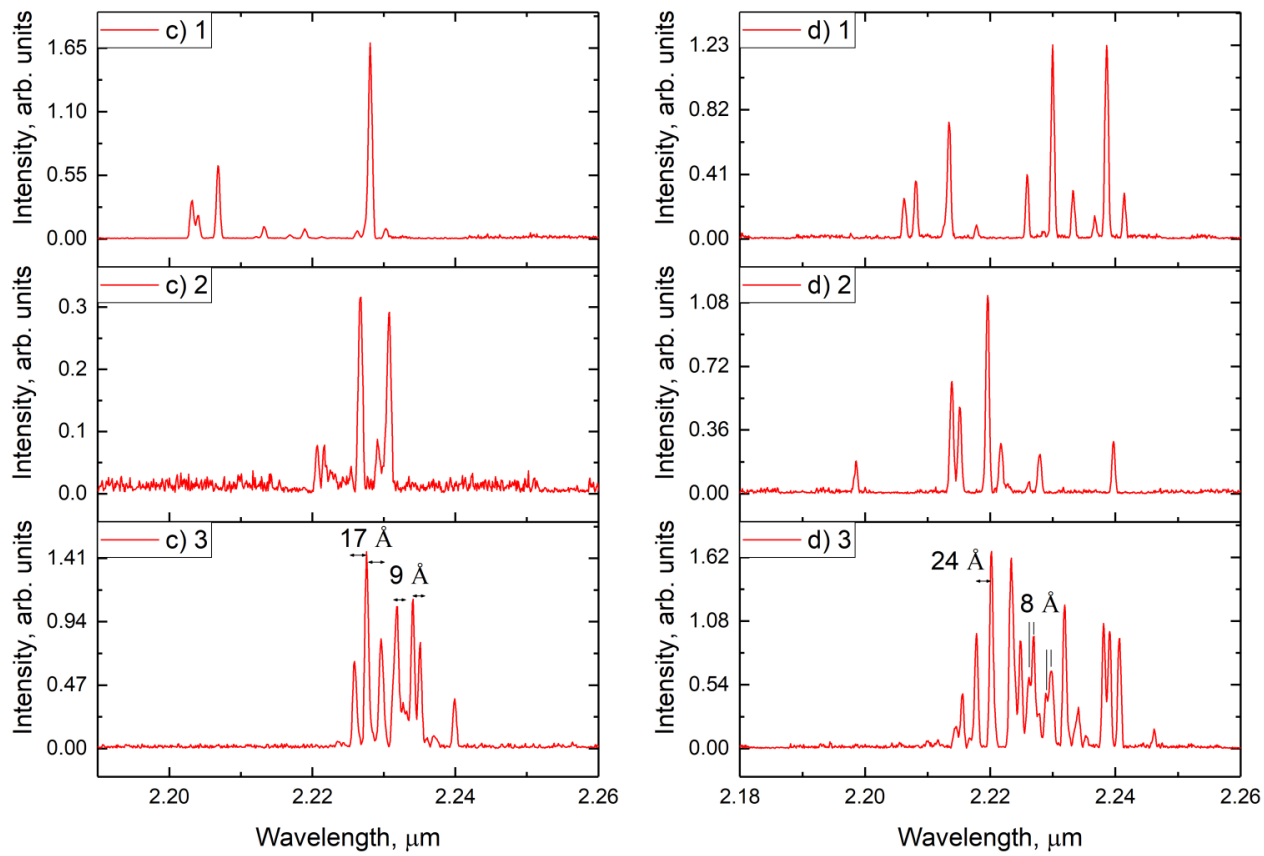

preferable for lasing in comparison to those from the first mode family, because the larger the effective mode area, the higher the optical amplification of the active media. The latter statement is being supported by our experimental results. In some cases, the generation of CMs is so beneficial that the system generates one the most efficient mode well above the laser threshold, which leads to a single mode operation. An example of the single mode operation can be seen in Fig. 4(b) 1, 2, 3 for a sample, which was given a name "sample 2." This sample has equal disk radii of $200 \mu \mathrm{m}$ and a conjunction that is $15 \mu \mathrm{m}$ wide and $10 \mu \mathrm{m}$ long. It is worth noting that the relative intensity of the single mode is stronger than the intensities of the modes of the separately pumped lasers, which 
Pumping current is fixed for the first cavity at $200 \mathrm{~A} / \mathrm{cm}^{2}$

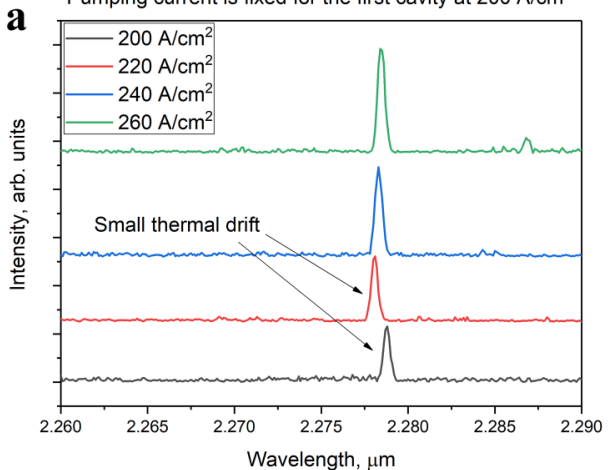

Pumping current is fixed for the second cavity at $200 \mathrm{~A} / \mathrm{cm}^{2}$

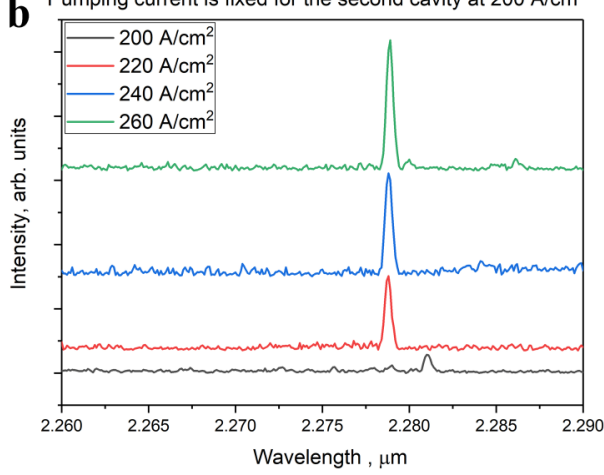

FIG. 5. Single mode operation of "sample 2" [shown in Fig. 5(b)] under different pumping conditions; (a) pumping current is fixed for the first cavity at $200 \mathrm{~A} / \mathrm{cm}^{2}$, while pumping current of the second cavity is being changed; (b) same for the second cavity. indicates the fact that most of the power goes to one single mode, while the other modes are suppressed [see Fig. 4(b)]. Interestingly, the single mode operation is not often observed in the strongly coupled WGM-laser samples, and it has been a topical subject in our research lately. Nevertheless, those samples that demonstrate the single mode operation are stable for changes in pumping currents even if we apply different currents to each cavity. Figure 5 shows how stable the single mode operation is to relative changes in pumping currents, and thus local temperatures in the cavities. If we fix the pumping current in one cavity to $200 \mathrm{~A} / \mathrm{cm}^{2}$ (it is well above the laser threshold, which is about $100-120 \mathrm{~A} / \mathrm{cm}^{2}$ for our lasers) and start to change the current in the other cavity, we will see that the relative changes do not affect the single mode operation at all [see Fig. 5(a)]. The same holds if we fix the pumping current for the second cavity [Fig. 5(b)]. This supports the idea that the single mode operation does not depend on relative positions of the resonant modes of the two cavities, but it is sensitive to their geometry, which is going to be discussed in more detail further in the text.

The major part of the laser samples with equal disk radii exhibit another operation regime, which is also referred to the collective mode family, since the mode structure changes drastically under the simultaneous pumping conditions. If we draw our attention to the emission spectra of one of these samples in the case when only one laser is pumped [see Fig. 4(c) 1,2], we will see that the mode structure is random, and there is no clear periodicity (or FSR) as it was in the previous cases. On the other hand, when we pump both lasers simultaneously [see Fig. 4(c) 3], the mode structure changes and the spectrum here is not just a sum of the spectra of two separately pumped lasers. Moreover, we observe a clear periodicity in the mode structure, and in some regions the mode spacing is less than $9 \AA$ for this particular sample [Fig. 4(c)], which is smaller than the FSR for a single disk laser with the same radius [12 A see Fig. 2(a)]. This sample (associated with a name "sample 3") is assumed to have the same geometry as the "sample 2 ," but the difference in operation is clear. We made an assumption that the difference could be associated with the asymmetry of the system, and in order to verify this idea we prepared another set of samples of strongly coupled WGM-lasers with deliberately sizemismatched disks. One can see the emission spectra of such a sample ("sample 4") in Fig. 4(d) 1, 2, 3 (see also Table I for the summary of all important parameters of the samples). This particular sample has highly size-mismatched disk radii of 100 and $85 \mu \mathrm{m}$ with $17 \mu \mathrm{m}$ wide and $10 \mu \mathrm{m}$ long conjunction, although we obtained the same results for larger disk radii (also size-mismatched) with approximately the same geometry of the conjunction. It is clear that the mode structure here resembles the one of "sample 3." Under the simultaneous pumping conditions, the mode structure demonstrates a typical multimode and periodic structure with unexpectedly narrow mode spacing ( $8 \AA$ in this case). Moreover, none of the sizemismatched samples exhibits a single mode operation, although the observed multimode operation can also be attributed to the generation of CMs, since these modes exist only under the simultaneous pumping conditions. We tested more than 30 intentionally size-

TABLE I. Summary of the most important parameters of the laser samples.

\begin{tabular}{|c|c|c|c|c|c|}
\hline Sample & Operation regime & Coupling type & Geometry & FEM figure & Spectra \\
\hline 1 & $\begin{array}{l}\text { Multimode, } \\
\text { Quasi-WGM }\end{array}$ & $\begin{array}{l}\text { Low coupling, almost no } \\
\text { interaction }<10 \mu \mathrm{m} \text { wide }\end{array}$ & $\begin{array}{l}\text { Assumed to be } \\
\text { size-matched }\end{array}$ & Fig. 3(d) & Fig. 4(a) \\
\hline 2 & Single mode, CMs & $\begin{array}{l}\text { Optimum coupling, medium } \\
\text { conjunction size } 15-20 \mu \mathrm{m} \text { wide }\end{array}$ & Size-matched & Fig. 3(c) & $\begin{array}{l}\text { Figs. 4(b), } \\
\text { 5(a), and 5(b) }\end{array}$ \\
\hline 3 & Multimode, CMs & $\begin{array}{l}\text { Optimum coupling, medium } \\
\text { conjunction size } 15-20 \mu \mathrm{m} \text { wide }\end{array}$ & $\begin{array}{l}\text { Unintentionally } \\
\text { size-mismatched }\end{array}$ & $\begin{array}{l}\text { N/A (disks were set to be } \\
\text { equal in the simulations) }\end{array}$ & Fig. 4(c) \\
\hline 4 & Multimode, CMs & $\begin{array}{l}\text { Optimum coupling, medium } \\
\text { conjunction size } 15-20 \mu \mathrm{m} \text { wide }\end{array}$ & $\begin{array}{c}\text { Intentionally } \\
\text { size-mismatched }\end{array}$ & $\begin{array}{l}\text { N/A (disks were set to be } \\
\text { equal in the simulations) }\end{array}$ & Fig. 4(d) \\
\hline
\end{tabular}


mismatched samples and the result is almost the same for all of them. Thus, one possible explanation here could be that due to the fabrication intolerances of the samples with equal radii the disks become unintentionally size-mismatched or asymmetric, which breaks prohibits the single mode operation. For that reason, in order to reliably fabricate the samples with the single mode operation one should optimize the etching process carefully.

\section{CONCLUSIONS}

To summarize, we demonstrated a special method of coupling for electrically pumped WGM-lasers. This method allows the optical modes to have optical paths that lie within both resonators without the need to leave the resonator. Moreover, we show that under certain circumstances, this type of coupling can lead to the generation of CMs and, in some special cases, to a single mode generation in a wide range of pumping currents. We performed a simulation using FEM, which shows the electromagnetic field distributions inside the laser cavities for two different cases, when the lasers are being pumped separately and simultaneously. Based on the simulations, we were able to classify the resonance modes of such systems. Furthermore, we demonstrate that under simultaneous pumping conditions, some resonance modes possess extremely complex electromagnetic field distributions. We attribute these modes to the collective mode family, confirming the possibility of generating them experimentally. Moreover, the generation of CMs is most likely the reason that leads to the single mode operation, although this type of operation is not often observed in the strongly coupled WGM-lasers so the multimode operation dominates. It was proposed that the problem of the single mode operation could lie in the asymmetry of the laser cavities. In order to shed some light on this problem, we conducted additional research with deliberately size-mismatched coupled WGM-lasers. Surprisingly, we found out that none of the size-mismatched samples demonstrated the single mode operation, whilst the multimode operation was still present as in the previous cases. Thus, the reliability of the single mode operation probably depends directly on the fabrication process, and it has to be optimized very carefully.

\section{ACKNOWLEDGMENTS}

This work is funded by the Russian Foundation for Basic Research (RFBR) (No. 18-52-00027 Bel_a). The authors wish to acknowledge Associate Professor Markku Vainio from the University of Helsinki for fruitful discussions.

\section{DATA AVAILABILITY}

The data that support the findings of this study are available from the corresponding author upon reasonable request.

\section{REFERENCES}

${ }^{1}$ V. S. Ilchenko and A. B. Matsko, "Optical resonators with whispering-gallery modes-Part II: Applications,” IEEE J. Sel. Top. Quant. 12(1), 15-32 (2006).

${ }^{2}$ L. He, S. Ozedemir, and L. Yang, "Whispering gallery microcavity lasers," Laser Photonics Rev. 7(1), 60-82 (2013).

${ }^{3}$ P. Del Haye, A. Schliesser, O. Arcizet, T. Wilkins, R. Holzwarth, and T. J. Kippenberg, "Optical frequency comb generation from a monolithic microresonator,” Nature 450, 1214-1217 (2008).
${ }^{4}$ A. N. Imenkov, V. V. Sherstnev, I. V. Kovalev, N. D. Il'inskaya, O. Yu. Serebrennikova, R. Teissier, A. N. Baranov, and Y. P. Yakovlev, "Temperature dependence of the threshold current in quantum-well WGM lasers (2.0-2.5 $\mu \mathrm{m})$," Semiconductors 47(6), 831-834 (2013).

${ }^{5}$ V. Sandoghdar, F. Treussart, J. Hare, V. Lefèvre-Seguin, J.-M. Raimond, and S. Haroche, "Very low threshold whispering-gallery-mode microsphere laser," Phys. Rev. A 54(3), R1777-R1780 (1996).

${ }^{6} \mathrm{~S}$. J. Herr, K. Buse, and I. Breunig, “Tunable single-frequency lasing in a microresonator," Opt. Express 27(11), 15351-15358 (2019).

${ }^{7}$ T. Beckmann, H. Linnenbank, H. Steigerwald, B. Sturman, D. Haertle, K. Buse, and I. Breunig, "Highly tunable low-threshold optical parametric oscillation in radially poled whispering gallery resonators," Phys. Rev. Lett. 106(14), 143903 (2011).

${ }^{8}$ F. Vollmer, S. Arnold, and D. Keng, "Single virus detection from the reactive shift of a whispering-gallery mode," Proc. Natl. Acad. Sci. U.S.A. 105(52), 20701-20704 (2008).

${ }^{\mathbf{9}}$ T. M. Benson, S. V. Boriskina, P. Sewell, A. Vukovic, S. C. Greedy, and A. I. Nosich, "Micro-optical resonators for microlasers and integrated optoelectronics," in Frontiers in Planar Lightwave Circuit Technology: Design Simulation and Fabrication, edited by S. Janz, J. Ctyroky, and S. Tanev (Springer, 2006).

${ }^{10} \mathrm{~S}$. V. Boriskina and L. Dal Negro, "Self-referenced photonic molecule bio (chemical)sensor," Opt. Lett. 35, 2496-2498 (2010).

${ }^{11}$ M. Bayer, T. Gutbrod, J. P. Reithmaier, A. Forchel, T. L. Reinecke, P. A. Knipp, A. A. Dremin, and V. D. Kulakovskii, "Optical modes in photonic molecules," Phys. Rev. Lett. 81, 2582-2585 (1998).

${ }^{12}$ S. V. Boriskina, "Spectrally engineered photonic molecules as optical sensors with enhanced sensitivity: A proposal and numerical analysis," J. Opt. Soc. Am. B 23, 1565-1573 (2006).

${ }^{13}$ O. Firstenberg, T. Peyronel, Q.-Y. Liang, A. V. Gorshkov, M. D. Lukin, and V. Vuletic, "Attractive photons in a quantum nonlinear medium," Nature 502, 71-75 (2013).

${ }^{14} \mathrm{~S}$. V. Boriskina, "Control of modes coupling, selection and enhancement in wavelength-scale optical microcavity structures: Applications to microlasers and biosensing," in Proceedings of IEEE 8th International Conference on Laser and Fiber-Optical Networks Modeling (IEEE, 2006), pp. 391-398.

${ }^{15}$ Q. Wang, H. Zhao, X. Du, W. Zhang, M. Qiu, and Q. Li, "Hybrid photonicplasmonic molecule based on metal/Si disks," Opt. Express 21(9), 11037-11047 (2013).

${ }^{16}$ Y. Li, F. Abolmaali, K. W. Allen, N. I. Limberopoulos, A. Urbas, Y. Rakovich, A. V. Maslov, and V. N. Astratov, "Whispering gallery mode hybridization in photonic molecules," Laser Photonics Rev. 11(2), 1600278 (2017).

${ }^{17}$ C. Schmidt, A. Chipouline, T. Käsebier, E.-B. Kley, A. Tünnermann, T. Pertsch, V. Shuvayev, and L. I. Deych, "Observation of optical coupling in microdisk resonators,” Phys. Rev. A 80, 043841 (2009).

${ }^{18} \mathrm{H}$. Wang, S. Liu, L. Chen, D. Shen, and X. Wu, "Dual-wavelength singlefrequency laser emission in asymmetric coupled microdisks," Sci. Rep. 6, 38053 (2016).

${ }^{19}$ D. A. Yarekha, G. Glastre, A. Perona, Y. Rouillard, F. Genty, E. M. Skouri, G. Boissier, P. Grech, A. Joullie, C. Alibert, and A. N. Baranov, "High temperature GaInSbAs/GaAlSbAs quantum well single-mode continuous wave lasers emitting near $2.3 \mu \mathrm{m}$," Electron. Lett. 36(6), 537-539 (2000).

${ }^{\mathbf{2 0}}$ A. Monakhov and N. Sablina, "Whispering gallery mode lasers," in Semiconductor Lasers Fundamentals and Applications, edited by A. Baranov and E. Tournie (Woodhead Publishing, 2013), Chap. 14.

${ }^{21}$ A. A. Dontsov, A. M. Monakhov, and N. S. Averkiev, "Calculation of the spectrum of whispering gallery modes in cylindrical resonators with perturbed boundary conditions," Quantum Electron. 43, 414-417 (2013).

${ }^{22}$ T. C. Chang, K. B. Hong, Y. Y. Lai, Y. H. Chou, S. C. Wang, and T. C. Lu, "ZnO-based microcavities sculpted by focus ion beam milling," Nanoscale Res. Lett. 11(1), 319 (2016).

${ }^{23}$ Q. Q. Duan, D. Xu, W. H. Liu, J. Lu, L. Zhang, J. Wang, Y. L. Wang, J. Gu, T. Hu, W. Xie, X. C. Shen, and Z. H. Chen, "Polariton lasing of quasi-whispering gallery modes in a ZnO microwire,” Appl. Phys. Lett. 24, 022103 (2013). 